\title{
Turbulent dynamos
}

\author{
B. Dubrulle ${ }^{1}$ \\ ${ }^{1}$ DRECAM/SPEC/CEA Saclay, and CNRS (URA2464), F-91190 Gif sur Yvette Cedex, France \\ email: berengere.dubrulle@cea.fr
}

\begin{abstract}
We discuss the problem of turbulent dynamo and illustrate it through numerical simulations and few results from the VKS experiment.
\end{abstract}

Keywords. MHD, Turbulence

\section{Introduction}

A great amount of the work of Juri concerns the solar dynamo, that converts turbulent convective motions into magnetic energy. It is described quite generally by the coupled set of equations:

$$
\begin{aligned}
\partial_{t} \mathbf{v}+(\mathbf{v} \cdot \nabla) \mathbf{v} & =-\frac{1}{\rho_{0}} \nabla P+\mathbf{j} \times \mathbf{B}+\nu \Delta \mathbf{v}+\mathbf{f}, \\
\partial_{t} \mathbf{B} & =\nabla \times(\mathbf{v} \times(\mathbf{B})+\eta \Delta \mathbf{B},
\end{aligned}
$$

where $\mathbf{v}$ is the fluid velocity, $P$ is the pressure, $\mathbf{B}$ is the Alfven velocity (or equivalently $\sqrt{\rho \mu_{0}} \mathbf{B}$ is the magnetic field), $j=\nabla \times \mathbf{B}$ is the magnetic current, $\nu$ and $\eta$ are the viscosity and the magnetic diffusivity, and $\rho_{0}$ is the (constant) fluid density. Four interesting dimensionless parameter are necessary to understand them: i) the Reynolds number $R e=L V / \nu$, where $L$ and $V$ are typical length and velocity; ii) the magnetic Reynolds number $R m=L V / \eta$ (or equivalently the Prandtl number $P m=R m / R e$ ); iii) the Rossby number $R o=U / L \Omega$, where $\Omega$ is the rotation rate (or equivalently the rotation number $\theta=1 / R o)$; iv) the interaction parameter $N=R m B^{2} / V^{2}$. It characterizes the ratio of the Lorentz force over the velocity advective term, and is a measure of the "non-linearity" of the MHD system: when $N \ll 1$, the magnetic field does not react back to the velocity field, and the two equations decouple.

In stars, both $R m$ and $R e$ are large. In numerical simulations, one can reach values of $R m$ up to 100 ( $P m$ varying from 1 to 0.01 ). In present days laboratory experiments with liquid metals, $R m$ cannot exceed 100, with $P m=2 \times 10^{-5}$. They are however quite interesting to understand the dynamo process in stars, as we discuss now.

\subsection{The VKS experiments}

The VKS experiment is based on stirring a von Karman flow of liquid sodium through impellers counter-rotating at frequencies $F_{1}$ and $F_{2}$. When monitoring the two frequencies, one can vary the rotation number $\theta=\left(F_{1}-F_{2}\right) /\left(F_{1}+F_{2}\right)$ between -1 and 1corresponding to regime of turbulence predominant over rotation. We have shown on Figure 1 the operating regime of the VKS experiments, to be compared with other natural objects. One sees that VKS operates in rotation number equivalent to that of sun-like-stars, but that another operating set up should be used to reach the parameters regime of the earth. This is interesting because most cosmic dynamos are turbulent, resulting in serious difficulties of modelling as we now discuss. 


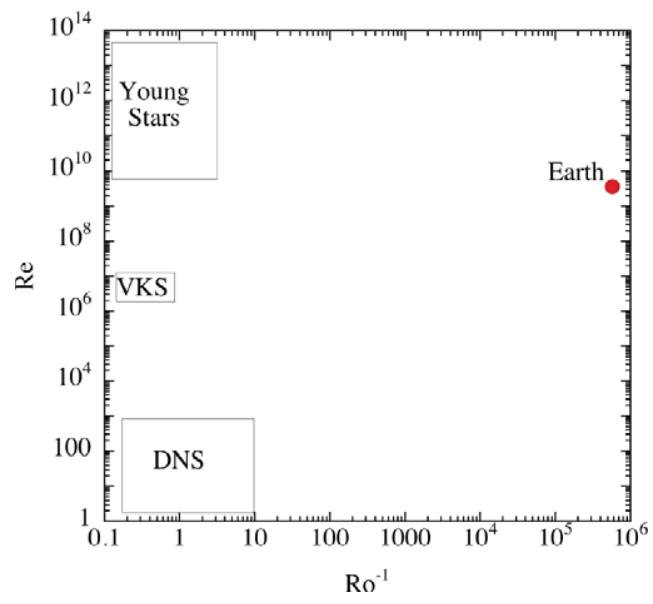

Figure 1. Typical range of Reynolds number and Rossby number for young stars, the earth, VKS2 experiment and DNS simulations.

\section{Laminar vs turbulent dynamo}

We first focus on the "linear" (also called "kinematic") case, where $N \ll 1$, so that $v$ can be considered as independent of $B$. We decompose the velocity field into a mean (time averaged) $\bar{U}$ and a fluctuating part $u^{\prime}$, with $\delta=<u^{\prime 2}>/<\bar{U}^{2}>$, the $<>$ denoting spatial average. Since the velocity field is independent of $B$, we can study the evolution of $B$ from the induction equations, that reads:

$$
\partial_{t} \mathbf{B}=\nabla \times(\overline{\mathbf{U}} \times \mathbf{B})+\nabla \times\left(\mathbf{u}^{\prime} \times \mathbf{B}\right)+\eta \Delta \mathbf{B} .
$$

This is a linear equation. Since $\bar{U}$ is by construction time-independent, it admits exponentially growing or decaying solutions in the absence of the second term of the r.h.s., like in any classical instability problem. The natural non-dimensional parameter to quantify the importance of the fluctuating term is $\epsilon=\delta-1$. Therefore, when $\epsilon \ll 1$, we have a "laminar" instability, with exponential growth or decay. The frontier in between the two behavior is the dynamo threshold, that will be close to the instability threshold computed only the mean flow.

For $\epsilon$ of order unity, the fluctuating term becomes important, and the equation now includes a time-dependent, stochastic like behavior. The instability is now akin to an instability in presence of a multiplicative noise, and requires special tools to be detailed later.

From the behavior of the parameter $\delta-1$ detailed in previous Section, we see that the dynamo is probably laminar for Taylor-Green flow at $R e<20$, or for VKS with an annulus and rotation, while it is probably turbulent for a TG flow with $R e>50$ and for VKS without an annulus.

\subsection{Laminar dynamo}

Laminar dynamo are countless. Some, like Ponomarenko or Robert's dynamo, can even be studied analytically. Here we focus on the TG and VKS laminar dynamos. In the case of the TG flow, the laminar dynamo is characterized by two windows of instability (Ponty et al. (2007); Laval et al. (2006)) (Fig. 2): the dynamo takes place for $R m_{c 1}<$ $R m<R m_{c 2}$ and for $R m>R m_{c 3}$. The three critical magnetic Reynolds number have been computed for mean flows measured at different Reynolds number $6<R e<100$ and 
were found to be roughly independent of Re. With the forcing adopted in Laval et al. (2006), one finds: $R m_{c 1} \sim 6, R m_{c 2} \sim 13$ and $R m_{c 3} \sim 25$.

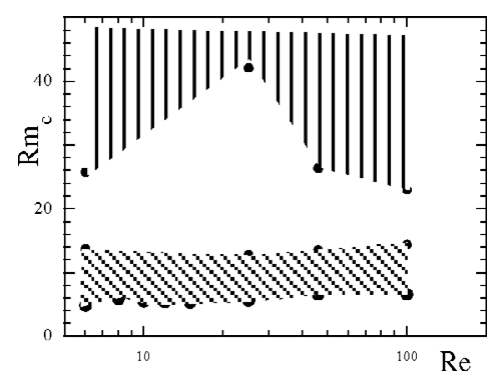

Figure 2. Windows of kinematic dynamo action with a time-averaged TG flow, as a function of the Reynolds number Re. The dashed area corresponds to region of the parameter space where kinematic dynamo is observed, corresponding to positive values of the Lyapunov exponent.

Because it was at the heart of VKS optimization, the laminar dynamo has been studied with different codes, and different boundary conditions or propeller shape and size. The lower threshold were obtained for TM73 propellers. In addition, it was found that the addition of a layer of sodium at rest produces a significant reduction of the dynamo threshold from $R m_{c} \sim 180$ to $R m_{c} \sim 40$ (Avalos-Zuniga et al. (2003); Ravelet et al. (2005)), and that the moving sodium behinds the propeller had a tendency to increase the dynamo threshold (Stefani et al. (2006); Laguerre et al. (20006)). This is summarized in Fig. 3.

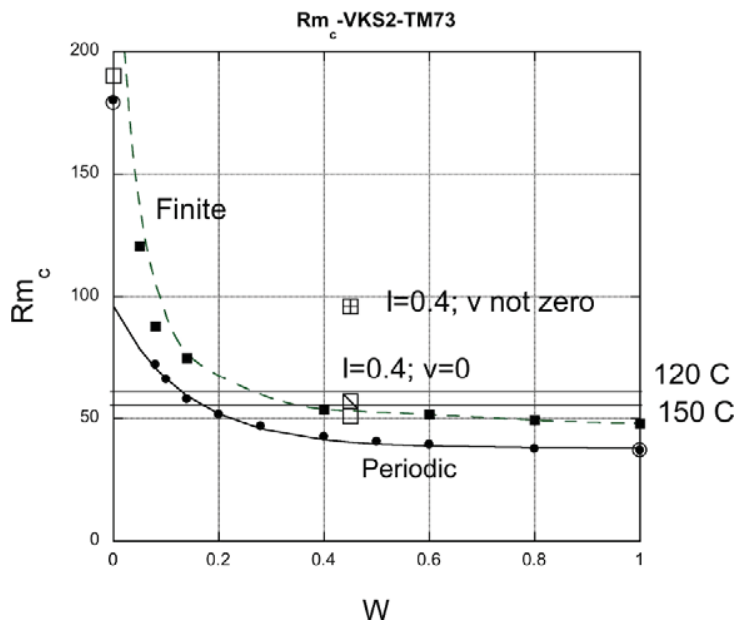

Figure 3. Critical value of the magnetic Reynolds number as a function of the percentage of sodium at rest $W$ from kinematic simulation with time-averaged von Karman flow with inox TM73 propellers rotating in the contra direction. Note that the kinematic simulation with iron propellers have not yet been done. Filled circle (Ravelet et al. (2005)) and open circle (courtesy C. Nore) : with periodic axial boundary conditions; Filled square : with finite axial boundary conditions (Laguerre et al. (20006)); open square (resp. square with cross): when taking into account the thin layer of fluid at rest (resp. stirred) behind the impellers (Stefani et al. (2006)). The two solid line delimit the largest $R m$ that can be reached in the VKS2 experiment at the lowest (resp. largest) operating temperature $120 \mathrm{C}$ (resp. $150 \mathrm{C}$ ). 
Specifically, the various threshold found with the kinetic simulation based on the timeaveraged velocity field with a layer of resting sodium of size $w=0.4$ are:

- $R m_{c}=43 \pm 1$ for periodic axial boundary conditions in a homogeneous conducting domain (Ravelet et al. (2005));

- $R m_{c}=49 \pm 2$ for finite axial boundary conditions;

- $R m_{c}=57$ (resp. 95) when taking into account the thin layer of fluid at rest (resp. stirred) behind the impellers (Stefani et al. (2006));

- $R m_{c}=46$ without the fluid behind the impellers for more realistic conditions: finite axial boundary condition, $5 \mathrm{~mm}$ copper shell separating the flow and the static conducting layer, copper container.

- $R m_{c}=55$ (resp. $R m_{c}=150$ ) for these conditions with the fluid behind the impellers at rest (resp. stirred). These results are given by Laguerre et al. (20006).

In the experiments, dynamo has been observed with iron propellers, with a threshold $R m_{c} \sim 32$ in contra rotation. With inox propellers, no dynamo has been observed in contrarotation. However, induction measurements with an external applied field $B_{a}$ can be used to estimate a dynamo threshold via the response $B_{i}$ as $B_{a} / B_{i} \sim \Lambda \sim R m-R m_{c}$. Linear fit to the induction curve $B_{a} / B_{i}$ then gives (see Fig. 4):

- $R m_{c}=127$ for TM73 inox propellers with no resting sodium and no annulus (VKS2b campaign);

- $R m_{c}=67$ for TM73 inox propellers with $w=0.4$ of resting sodium and no annulus (VKS2a campaign);

- $R m_{c}=53$ ) for TM73 inox propellers with $w=0.4$ of resting sodium and an annulus (VKS2f campaign);

- $R m_{c}=32$ for TM73 iron propellers with $w=0.4$ of resting sodium and an annulus (VKS2g campaign).

The decrease of $R m_{c}$ seen between $2 \mathrm{~b}$ and $2 \mathrm{a}$ suggests that indeed the resting sodium is favorable to dynamo action. The difference between $2 \mathrm{a}$ and $2 \mathrm{f}$ threshold suggests that the turbulence (described by the parameter $\delta$ ) has an impact on the dynamo threshold. This is the subject of the next section.

\subsection{Turbulent dynamo}

We consider now a situation where fluctuation are non-negligible. A first natural approach is to identify a small parameter $\epsilon$ in the problem, and try and solve the full problem by perturbation theory. Specifically, one consider first the time-averaged of Eq. (2.1):

$$
\partial_{t} \overline{\mathbf{B}}=\nabla \times(\overline{\mathbf{U}} \times \overline{\mathbf{B}})+\nabla \times\left(\overline{\mathbf{u}^{\prime} \times \mathbf{b}^{\prime}}\right)+\eta \Delta \overline{\mathbf{B}} .
$$

The main idea is to find the shape of $\overline{\mathbf{u}^{\prime} \times \mathbf{b}^{\prime}}$ as a function of $\bar{B}$ through the perturbation expansion.

An historically successful approach is to consider an ideal case where there is a scale separation between the typical scale $l$ of $\left(u^{\prime}, b^{\prime}\right)$ and the typical scale $L$ of $\bar{B}$. The natural expansion parameter is therefore $\epsilon=l / L \ll 1$, or equivalently $\nabla \bar{B}$. Without any computations, one can then infer that

$$
\epsilon_{i j k} \overline{u_{j}^{\prime} \times b_{i}^{\prime}}=\alpha_{i j} \overline{B_{j}}+\beta_{i j k} \nabla_{j} \overline{B_{k}}+O\left(\epsilon^{2}\right),
$$

where $\alpha_{i j}$ and $\beta_{i j k}$ are two tensors that depend on the velocity field and that can be computed through classical perturbation procedure applied to Eq. (2.1) (see e.g. Dubrulle \& Frisch (1991)). When plugged back into (2.2), this expansion gives:

$$
\partial_{t} \overline{\mathbf{B}}=\nabla \times(\overline{\mathbf{U}} \times \overline{\mathbf{B}})+\nabla_{j} \alpha_{i j k} \overline{\mathbf{B}_{\mathbf{k}}}+\nabla_{j} \nabla_{k}\left(\beta_{i j k l}+\eta \delta_{j k} \delta_{i l}\right) \overline{\mathbf{B}_{\mathbf{l}}},
$$



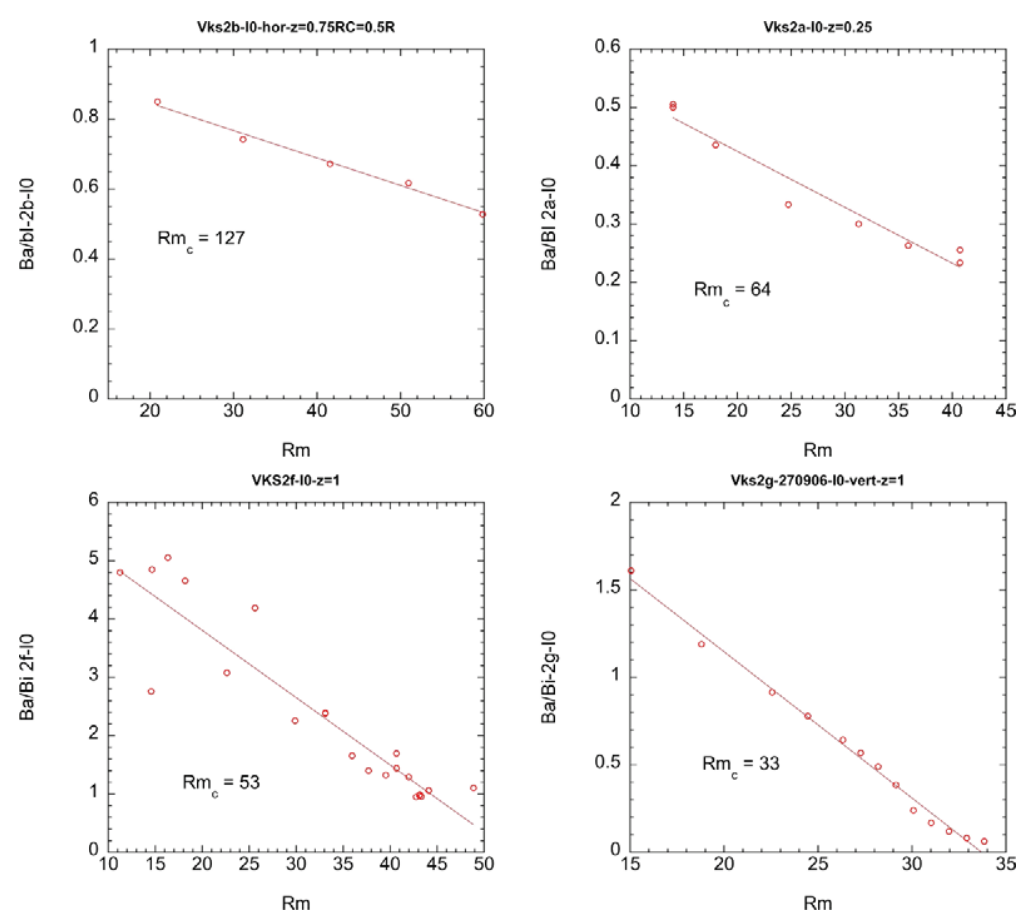

Figure 4. Estimate of dynamo threshold from induction measurements in VKS2 through the quantity $B_{a} / B_{i}$ in four different configuration : Upper left : $2 \mathrm{~b}$ : no resting sodium, TM73 inox propellers, no annulus; Upper right : $2 \mathrm{a}: 40 \%$ of resting sodium, TM73 inox propellers, no annulus; Lower left : $2 \mathrm{f}$ : $40 \%$ of resting sodium, TM73 inox propellers, annulus; Lower right : $2 \mathrm{~g}: 40 \%$ of resting sodium, TM73 iron propellers, annulus. The lines are linear fit, providing the value of $R m_{c}$ indicated on each plot.

where $\alpha_{i j k}=\epsilon_{i j m} \alpha_{m k}$ and $\beta_{i j k l}=\epsilon_{i k m} \beta_{m j l} . \alpha$ is the famous alpha coefficient, while $\beta$ is a turbulent diffusivity tensor, that need not be definite positive. In the absence of mean flow, this equation usually leads to a large scale instability via the alpha effect.

\section{Stochastic Theory}

Non-perturbation computations can be performed both analytically and numerically by replacing the true velocity fluctuations by some well chosen noise. Of course, real turbulence is characterized by temporal and spatial correlation that cannot be captured by such a simple noise. One can however hope that first order effects can be captured by our simple model. The reader can judge by himself from the final comparison. In any case, the advantage of these stochastic computations is twofold: first, they allow for non-perturbation analytical and numerical computations; second, their numerical cost is equivalent to the cost of a kinematic simulation. Simulation of $64^{3}$ can then prove sufficient to explore a range of fluctuations equivalent to $R e=10^{7}$, (i.e. that would require $10^{15}$ grid points).

We therefore now consider that $u^{\prime}$ is a white noise, characterized by:

$$
\left\langle u_{i}^{\prime}(x, t) u_{j}^{\prime}\left(x^{\prime}, t^{\prime}\right)\right\rangle=2 G_{i j}\left(x, x^{\prime}\right) \delta\left(t-t^{\prime}\right),
$$

where the brackets denote ensemble average, over the realizations of the noise. Equation (2.1) then takes the shape of a stochastic partial differential equation for $B$, with multiplicative noise. The problems associated with this type of noise can be understood by 
looking at a simple unidimensional model:

$$
\dot{x}=\mu x+\xi x,
$$

where $\xi(t) \xi\left(t^{\prime}\right)=2 D \delta\left(t-t^{\prime}\right)$. In the absence of noise, $x$ is exponentially increasing (unstable) as soon as $\mu>0$. In the presence of noise, we can take different moments of the equation and get the following hierarchy:

$$
\begin{aligned}
<\dot{x}> & =(D+\mu) \\
<\dot{x}^{2}> & =2(2 D+\mu)
\end{aligned}
$$

so that the $\langle x\rangle$ (resp. $\left\langle x^{2}\right\rangle$ ) is unstable for $\mu>-D$ (resp. $\mu>-2 D$ ). Therefore, its seems that the instability threshold depends on the moment we consider! One can in fact prove that this pathology is due to the absence of non-linear terms, and that in fact the correct threshold that would prevail with non-linear term is captured by considering the Lyapunov:

$$
\Lambda=\partial_{t}<\ln x>\text {. }
$$

Due to the convexity of the $\log , \Lambda \leqslant \partial_{t} \ln \langle x\rangle$, so that $\Lambda$ is always smaller than the growth rate. The system is unstable as soon as $\Lambda>0$.

Analytical computation of the stochastic model have been done by Leprovost \& Dubrulle (2005). In order to make the computations tractable, two approximation were made: i) a saturating term was added to the induction equation as $-c B^{2} B_{i}$ because of symmetry consideration. In some sense, this modification is akin to an amplitude equation, and the cubic shape for the non-linear term could be viewed as the only one allowed by the symmetries. Such a procedure is motivated by the observation that the precise form of the nonlinear term does not affect the threshold value. ii) The diffusivity was ignored.

Using standard techniques (Boldyrev (2001)), one can then derive the evolution equation for $P(\mathbf{B}, x, t)$, the probability of having the field $\mathbf{B}$ at point $x$ and time $t$ :

$$
\begin{aligned}
\partial_{t} P= & -\bar{U}_{k} \partial_{k} P-\left(\partial_{k} \bar{U}_{i}\right) \partial_{B_{i}}\left[B_{k} P\right]+\partial_{k}\left[\beta_{k l} \partial_{l} P\right] \\
& +c \partial_{B_{i}}\left[B^{2} B_{i} P\right]+2 \partial_{B_{i}}\left[B_{k} \alpha_{l i k} \partial_{l} P\right] \\
& +\mu_{i j k l} \partial_{B_{i}}\left[B_{j} \partial_{B_{k}}\left(B_{l} P\right)\right]
\end{aligned}
$$

with the following turbulent tensors:

$$
\beta_{k l}=\left\langle u_{k}^{\prime} u_{l}^{\prime}\right\rangle, \quad \alpha_{i j k}=\left\langle u_{i}^{\prime} \partial_{k} u_{j}^{\prime}\right\rangle \quad \text { and } \quad \mu_{i j k l}=\left\langle\partial_{j} u_{i}^{\prime} \partial_{l} u_{k}^{\prime}\right\rangle
$$

Due to incompressibility, the following relations hold: $\alpha^{k i i}=\mu^{i i k l}=\mu^{i j k k}=0$.

The physical meaning of these tensors can be found by analogy with the "Mean-Field Dynamo theory" (Krause\& Rädler (1980); Moffatt (1978)). Indeed, consider the equation for the evolution of the mean field, obtained by multiplication of equation (3.5) by $B_{i}$ and integration:

$$
\begin{aligned}
\partial_{t}\left\langle B_{i}\right\rangle= & -\bar{U}_{k} \partial_{k}\left\langle B^{i}\right\rangle+\left(\partial_{k} \bar{U}_{i}\right)\left\langle B_{k}\right\rangle-2 \alpha_{k i l} \partial_{k}\left\langle B_{l}\right\rangle \\
& +\beta_{k l} \partial_{k} \partial_{l}\left\langle B_{i}\right\rangle-c\left\langle B^{2} B_{i}\right\rangle .
\end{aligned}
$$

This equation resembled the classical Mean Field Equation of dynamo theory, with generalized (anisotropic) " $\alpha$ " and " $\beta$ ". The first effect leads to a large scale instability for the mean-field, while the second one is akin to a turbulent diffusivity. Note that the tensor $\mu$ does not appear at this level. 
The Lyapunov exponent can be computed in a similar way from (3.5) by changing variable $B_{i}=B e_{i}$, then multiplying the resulting equation by $B^{d-1} \ln B$ and integrating with respect to $B$. This yields:

$$
\Lambda \equiv \partial_{t}\langle\ln B\rangle=\left\langle\partial_{k} \bar{U}_{i} e_{i} e_{k}\right\rangle_{\phi}+\left\langle\mu_{i j k l}\left(\Delta_{i k} e_{j} e_{l}+\Delta_{k j} e_{i} e_{l}\right)\right\rangle_{\phi},
$$

where we used $\Delta_{i j}=\partial_{n_{i}}\left(n_{j}\right)=\delta_{i j}-e_{i} e_{j}$ an "angular Dirac tensor", and the symbol $\langle\bullet\rangle_{\phi}$ denotes verages over the angular variables.

The condition for dynamo action in this model is $\Lambda>0$. In the limit of zero noise, the term proportional to $\mu$ is negligible and one recovers the classical criterion for instability in a laminar dynamo in the infinite Prandtl number limit. Indeed, in such a case, the magnetic field will mainly grow in the direction given by the largest eigenvalue $\lambda_{\max }$ of $S_{i j}=\partial_{j} \bar{U}_{i}$, so that

$$
\Lambda \approx\left\langle\partial_{k} \bar{U}_{i} e_{i} e_{k}\right\rangle_{\phi}=\lambda_{\max }
$$

There will be dynamo only if $\lambda_{\max }>0$.

Consider now a situation where you increase the noise level. Two different influences on the sign of $\Lambda$ then result: one through the factor proportional by $\mu$. According to the sign of this factor, it can therefore favor or hinder the dynamo. In isotropic homogeneous turbulence, $\mu$ is positive, so that it is in general favorable to dynamo action. Moreover, being proportional to derivatives of the noise, this term gets larger as the typical scale of the noise is small.

However, there exists another less obvious -and adverse- influence of the noise: the disorientation effect. Indeed, noise changes the distribution of magnetic field orientation. In the absence of noise, the latter tends to be oriented towards the most unstable direction. However, noise constantly drives the magnetic field away from this favorable direction, sometimes even driving it towards a stable direction, where the magnetic field exponentially decreases. The net result is a decrease of the effective growth rate of the magnetic field. A phenomenological way to quantify this effect is through the parameters $\delta-1$ and $\delta_{2}$. Indeed, the largest these coefficient are, the further away the instantaneous velocity field is from the averaged field, and the largest the disorientation effect can be. This effect is more important when the noise it at largest scale, since in that case the disorientation effect is more pronounced-one can get farther from the mean flow.

From this discussion, one expects large scale noise to be adverse to dynamo actionthrough the disorientation mechanism, while small scale noise should be favorable to dynamo action-through the $\mu$ effect.

The previous analytical computation were tractable only in the limit $\eta \rightarrow 0(R m \rightarrow \infty$. To investigate the more realistic case of finite diffusivity, one may resort to numerical simulations. This has been done by Laval et al. (2006) and Dubrulle et al. (2007) for the case of the Taylor-Green flow, without inclusion of the non-linear term in the induction equation. Two kinds of noise were tested: shortly correlated noise, like in the analytical case, and Markovian noise, with finite correlation time $\tau_{c}$ that can be varied from 0 to several eddy-turnover time. Two typical noise scale were also tested, one at the largest available scale of the system $k=1$, and one of the order of the magnetic diffusive scale $k=16$.

The results are summarized in Fig. 5 for time-correlated noises at large and small scale. In the case of the large scale noise, one sees that the two dynamo windows are lifted up by the noise, resulting in an increase of the dynamo threshold. It can also be shown that this effect becomes more pronounced as the correlation time of the noise increase until the mean eddy turn over time is reached. Above this, the effect does not change anymore. From the previous discussion, we can attribute this increase of the threshold 

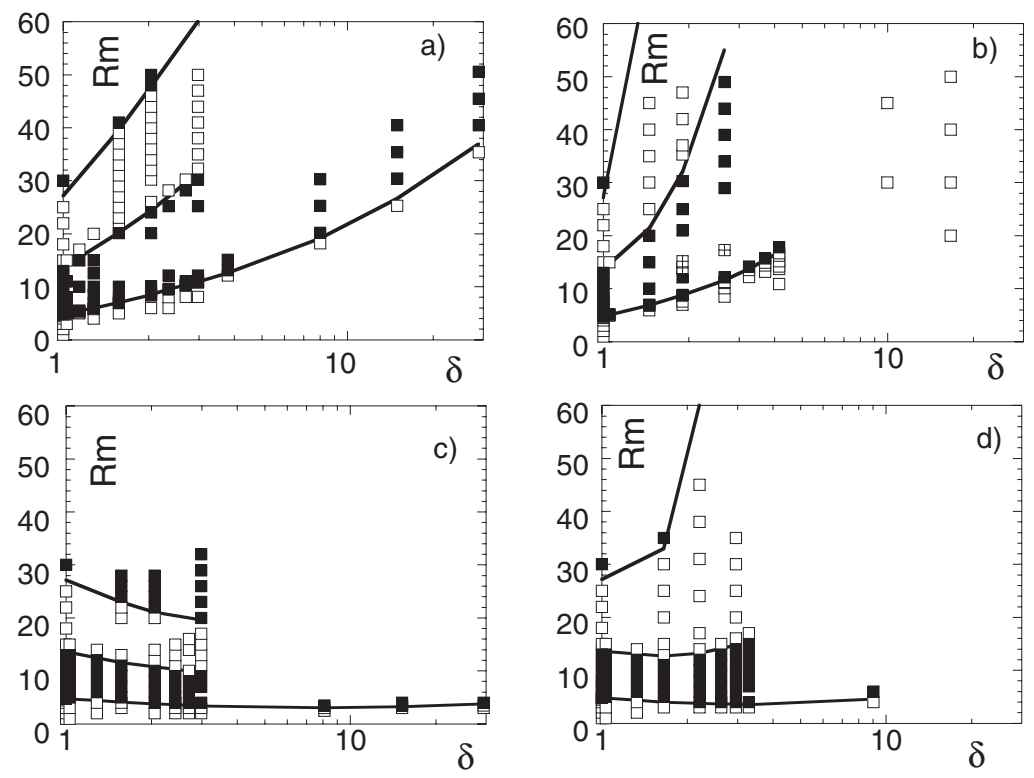

Figure 5. Parameter space for noise at $R e=6$ for different noise parameters : a) $\tau_{c}=0$, $k_{I}=1$; b) $\tau_{c}=0.03, k_{I}=1$; c) $\tau_{c}=0, k_{I}=16$; d) $\tau_{c}=0.03, k_{I}=16$. Open square : no-dynamo case; Square with cross : undecided state; Filled square : dynamo case. The full lines are zero-Lyapunov lines.

to the disorientation effect. In contrast, when the noise it at small scale, the dynamo threshold is -slightly- decreased with respect to the laminar case. This is probably a benefit of the $\mu$ effect.

The influence of the noise onto the first dynamo threshold can be summarized by plotting the critical magnetic Reynolds numbers as a function of the noise intensity (Fig. 6-a). Large scale (resp. small-scale) noise tends to increase (resp. decrease) the dynamo threshold. For small noise intensities, the correction $R m_{c}^{t u r b}-R m_{c}^{M F}$ is linear in $\delta-1$, in agreement with the perturbation theory (Pétrélis (2002)). Furthermore, one sees that for small scale noise, the decrease in the dynamo threshold is almost independent of the noise correlation time $\tau_{c}$, while for the large scale noise, the increase is proportional to $\tau_{c}$ at small $\tau_{c}$. At $\tau_{c}>1$, all curves $R m_{c}(\delta)$ collapse onto the same curve. We have further investigated this behavior to understand its origin. Increasing $\delta$ first increases of the flow "turbulent viscosity" $\overline{v_{r m s}} \overline{l_{i n t}}$ with respect to its mean flow value $V_{r m s} L_{i n t}$. This effect can be corrected by considering $R m_{c}^{*}=R m_{c} V_{r m s} L_{i n t} / \overline{v_{r m s}} \overline{l_{i n t}}$. Second, an increase of $\delta$ produces an increase of the fluctuations of kinetic energy, quantified by $\delta_{2}$. This last effect is more pronounced at $k_{I}=1$ than at $k_{I}=16$. It is amplified through increasing noise correlation time. We thus re-analyzed our data by plotting $R m_{c}^{*}$ as a function of $\delta_{2}$ (Fig. 6-b). All results tend to collapse onto a single curve, independently of the noise injection scale and correlation time. This curve tends to a constant equal to $R m_{c}^{M F}$ at low $\delta_{2}$. This means that the magnetic diffusivity needed to achieved dynamo action in the mean flow is not affected by spatial velocity fluctuations. This is achieved for small scale noise, or large scale noise with small correlation time scale. In contrast, the curve diverges for $\delta_{2}$ of the order of 0.2 , meaning that time-fluctuations of the kinetic energy superseding 20 percent of the total energy annihilate the dynamo.

An obvious stationary solution of (3.5) is a Dirac function, representing a solution with vanishing magnetic field. Another stationary solution can be found for $B$ such that 

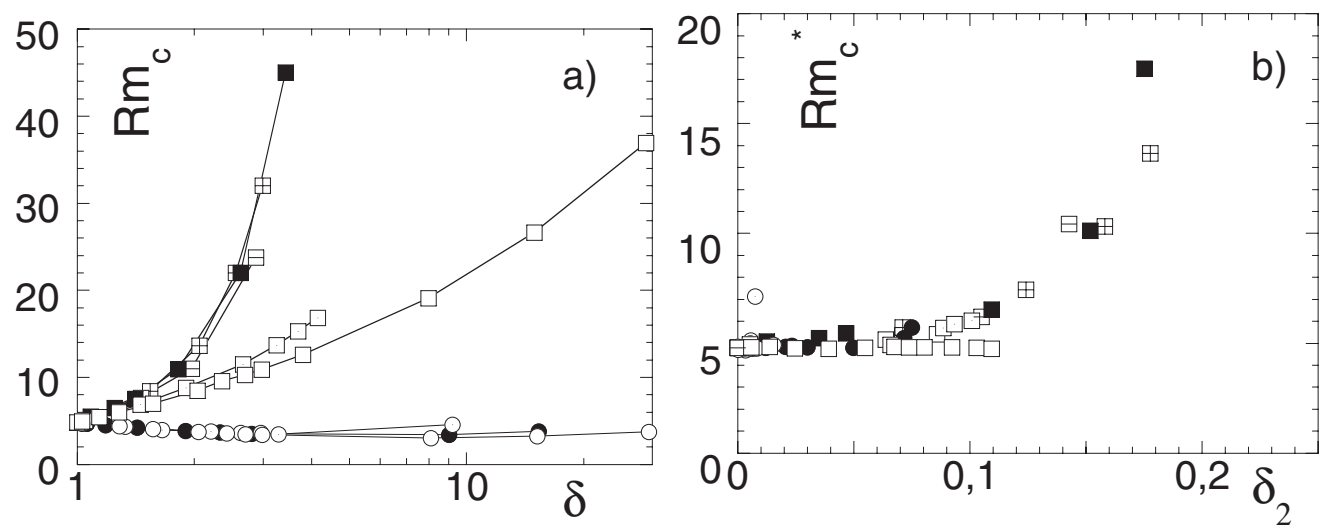

Figure 6. Evolution of the dynamo threshold for KS simulations with $\bar{u}(R e=6)$. a) $R m_{c}$ as a function of $\delta$ and b) $R m_{c}^{*}$ as a function of $\delta_{2}$ for different noise parameters : $k=1$ : square $\tau_{c}=0$; boxdot : $\tau_{c}=0.1 \mathrm{sec}$; boxminus : $\tau_{c}=1 \mathrm{sec}$; boxplus : $\tau_{c}=8 \mathrm{sec}$; black square $\tau_{c}=50$ sec; $k=16$ : circle : $\tau_{c}=0$; odot $: \tau_{c}=0.1 \mathrm{sec}$; bullet $: \tau_{c}=50 \mathrm{sec}$.

$B_{i}=B e_{i}$ by setting $\partial_{t} P=0$ in (3.5), with solution:

$$
P(B)=\frac{1}{Z} B^{\Lambda / a-1} \exp \left[-\frac{c}{2 a} B^{2}\right],
$$

where $Z$ is a normalization constant and $a=\left\langle\mu_{i j k l} e_{i} e_{j} e_{k} e_{l}\right\rangle_{\phi}$. This solution can represent a meaningful probability density function-and therefore a dynamo case- only if it can be normalized. Condition of integrability at infinity of (3.10) requires $a$ be positive. This illustrates the importance of the non-linear term which is essential to ensure vanishing of the probability density at infinity. Condition of integrability near zero requires $\Lambda>0$ be positive.
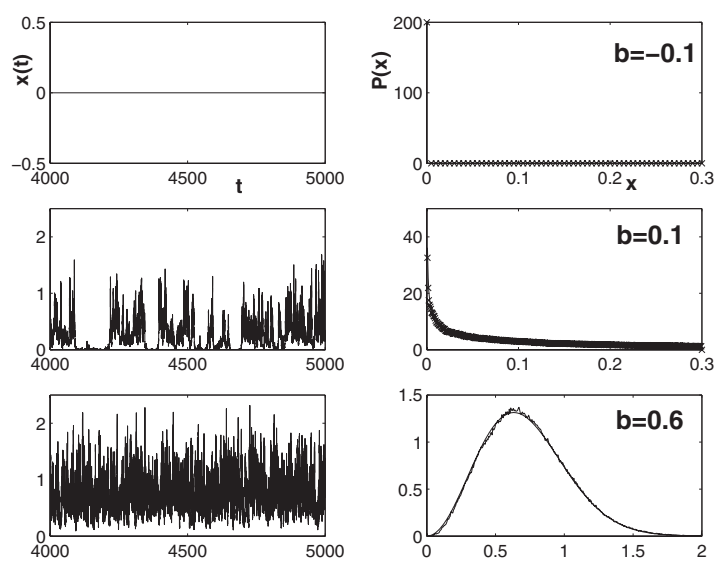

Figure 7. Result of the surrogate 1D model $\partial_{t} x=[b+\xi(t)] x-\gamma x^{3}$ : On the left side we show time series for $a=0.2, \gamma=1$ and 3 different values of the parameter $b$. On the right side, the corresponding PDF and the theoretical curve corresponding to equation (3.10), with $\Lambda=b$.

This is the dynamo condition identified before, that is obtained using the mean field as control parameter. However, the shape of the PDF traces an interesting new paradigm for the turbulent dynamo (Fig. 7). Indeed, in the range $0<\Lambda<a$, the PDF is maximum at zero, meaning that the most probable value for the magnetic field is zero. This is 
the signature of an "intermittent" dynamo, with periods of large magnetic field followed by quiescent periods, in a way reminiscent to "on-off" intermittency. Above this second threshold, $\Lambda>a$, the PDF exhibits a non-zero value for its most probable value, meaning a more classical "turbulent stationary dynamo", with fluctuations of the magnetic field around a finite value. Note that the transition from one regime to another can be mediated by the value of $\delta-1$ : as this parameter is increased, the disorientation effect becomes more and more important, and $\Lambda$ decreases. This remark is corroborated by recent stochastic computations of Aumaitre et al. (2005), who showed that the intermittent behavior could be switched off by changing the value of the noise spectrum at zero frequency, i.e. by removing large scale noise. Note also that this new paradigm cannot be tested in the previous TG computations, since they did not include any non-linearities. A more serious question is also: Is this new paradigm an artifact of our synthetic turbulence, or is it something that one can actually see? To check this, one needs to resort to numerical simulations or experiments.

\section{Taylor-Green Numerical simulations}

Direct numerical simulation of Eq. (1.1) for Taylor-Green forcing have been made in Ponty et al. (2005), Laval et al. (2006), Ponty et al. (2007) and Dubrulle et al. (2007). The dynamo threshold $R m_{c}$ has been computed for different values of $R e$. The result is shown in Fig. 8. One sees that the dynamo threshold increases with $R e$ until a value of the order $R e \sim 100$ where it seems to saturate. Dubrulle et al. (2007) also performed computations at low $R e$ but larger and larger $R m$ to try and detect a possible signature of the second laminar window of instability. At $R e=6$, they detected a transition from an intermittent dynamo at $R m=25$, to a dynamo with a mean field at $R m=100$ (see Fig. 9). Moreover, one can see a remarkable correlation between the dynamo windows predicted by the stochastic numerical simulation and the direct numerical simulation. This is an indication that maybe the stochastic model does capture the main features of the turbulent dynamo transition.

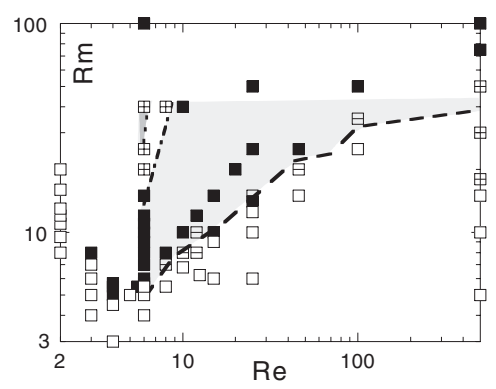

Figure 8. Comparison between DNS and KS simulations with $R e=6$ with $k_{I}=1, \tau_{c} \geqslant 0.3$. Squares refer to DNS-MHD and LES-MHD simulations, and shaded areas to windows of dynamo action for kinematic-stochastic simulations at $R e=6$ with $k_{I}=1, \tau_{c} \geqslant 0.3$. Note the tiny dynamo window near $R e=6, R m=40$. Open square : no-dynamo case; Square with cross : intermittent dynamo; Filled square : dynamo case; square with line : undecided state; $-R m_{c}^{\text {turb }}$; $--R m_{c}^{M F} ;-\cdot-$. end of the first dynamo window; $\cdots$ beginning of the second dynamo window.

\section{Experiments}

Various configurations have been tested in VKS with the TM73 propellers and the layer of sodium at rest: with and without annulus, and with inox or iron propellers. In the inox 

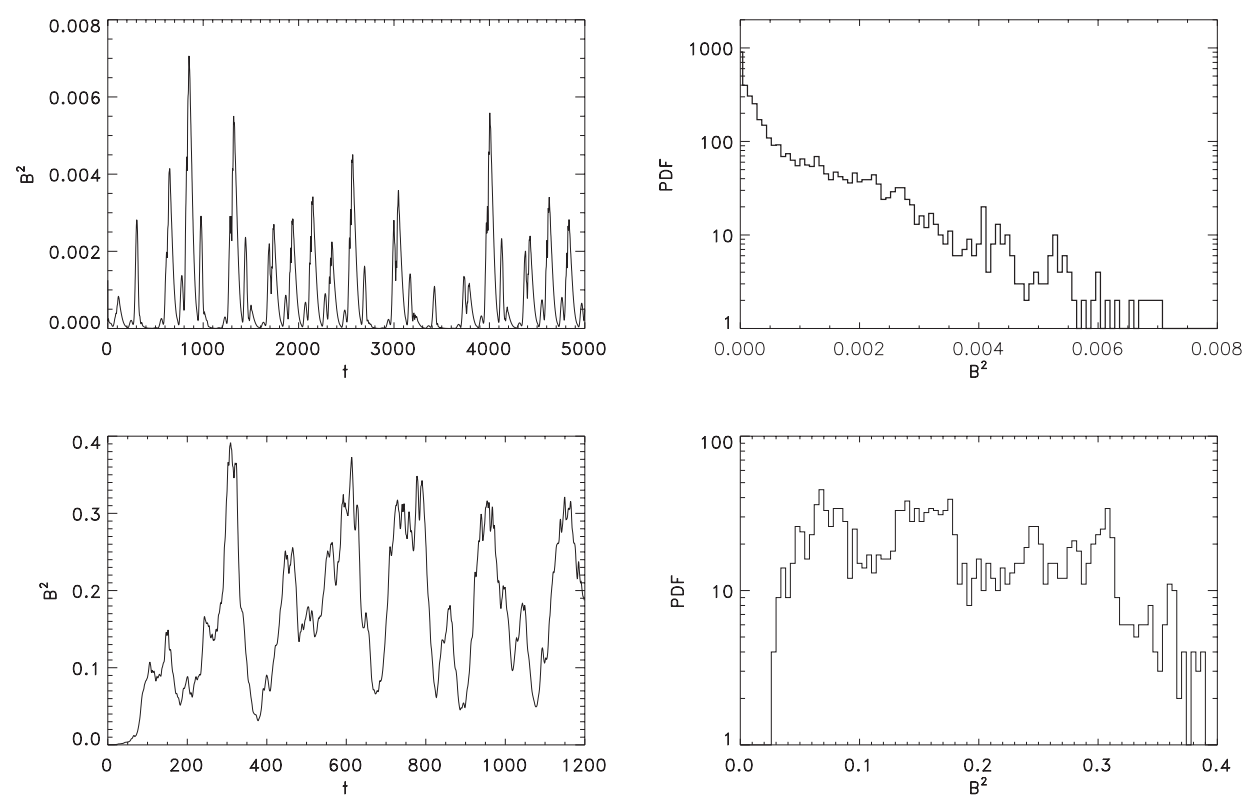

Figure 9. Example of dynamos in TG flow. Left : Magnetic energy as a function of time. Right : PDF of the magnetic energy. Upper panel : Intermittent dynamo $R e=6, R m=40$. Lower panel : Turbulent dynamo $R e=25, R m=50$.

case, no dynamo has been observed (Ravelet et al. (2008)). However, a critical magnetic Reynolds number could be estimated from induction measurements for configurations with positive and negative rotation. In the induction regime, the disorientation effect could be directly measured by following strong local magnetic field perturbations (Volk et al. (2006)).

In the iron case, with an annulus in the midplane, different types of dynamo have been identified, in the rotating and non-rotating case (Berhanu et al. 2007; Monchaux et al. (2007); Monchaux et al. (2008)). Among them, intermittent dynamo have been observed around $\theta=0.2$ (see Fig. 10).
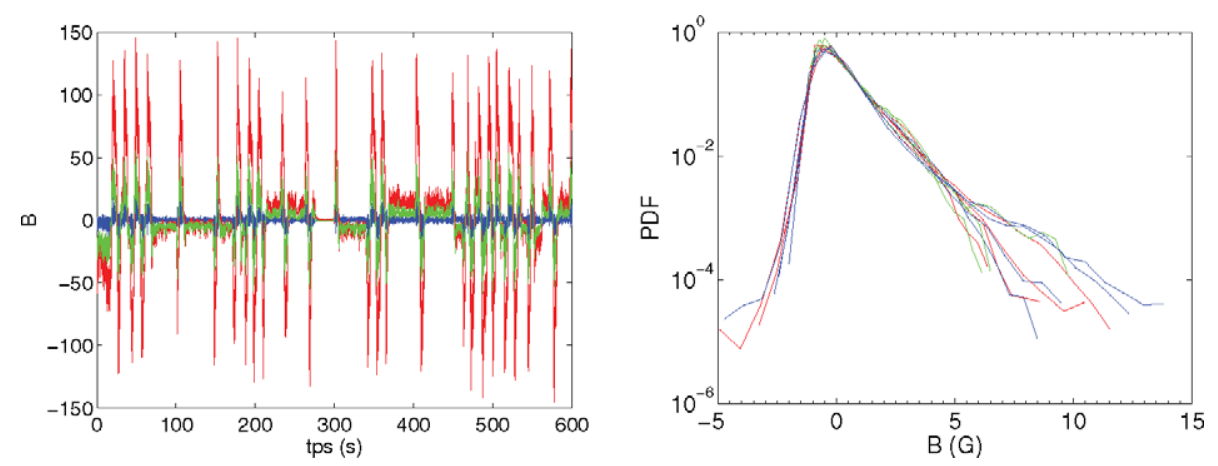

Figure 10. Example of intermittent dynamo observed in the VKS2 with TM73 iron propellers at $\theta=0.17, R m=32$. Left : Components of the magnetic field as a function of time (red : $B_{z}$, green $B_{\theta}$; blue : $B_{r}$. Right : Corresponding PDF of the magnetic field components.

Note that the intermittent dynamo observed near $\theta=0.2$ is characterized by the largest 
value of $\delta$. In the previous Section we argued that it was probably a good condition to observe, if any, the intermittent dynamo.

Regarding threshold for dynamo instability (transition towards stationary dynamo), it has been accurately measured so far in 3 cases: at $\theta=0$, with impellers rotating in the $(+)$ or $(-)$ direction with respect to the pales curvature; at $\theta=-1$ with impellers rotating in the $(+)$ direction. Using the values of $\delta$ measured in water, we can check whether the trend observed in TG numerical simulation (higher threshold for larger values of $\delta$ or $\delta_{2}$, see Fig. 6) is also valid here. With the presently available data, the trend is indeed respected (Fig. 11, but more data is needed to confirm this point.
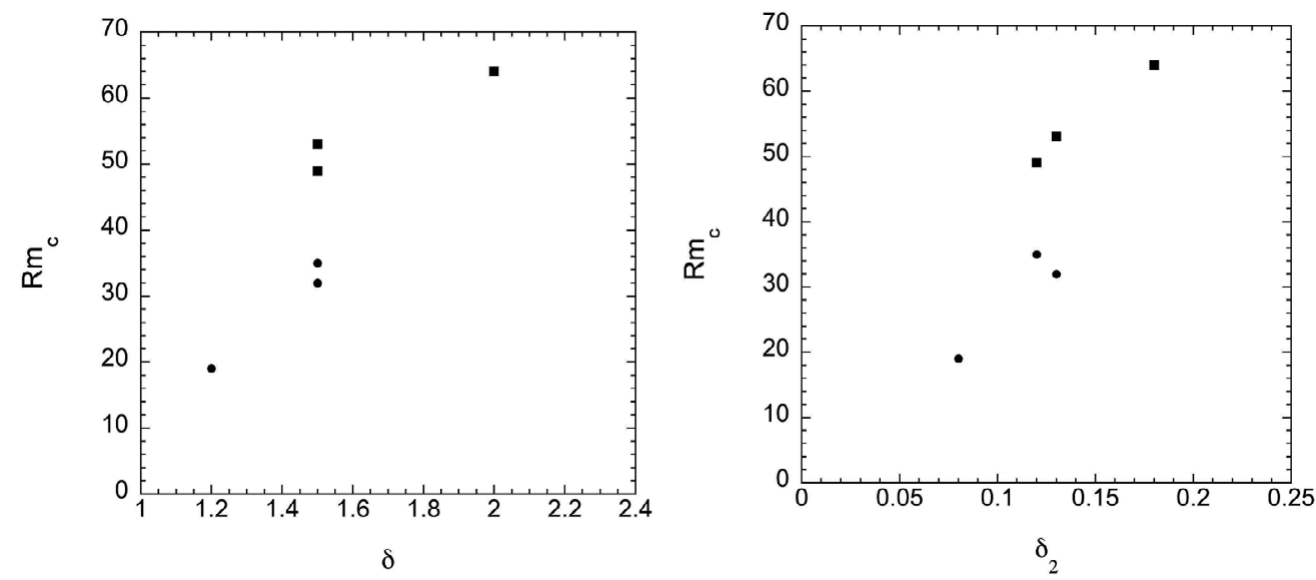

Figure 11. Critical magnetic Reynolds number $R m_{c}$ as a function of $\delta$ (left) and $\delta_{2}$ (right) in the VKS2 experiment for inox (square) and iron propellers ( circle). The $\delta_{2}$ have been estimated from the water model experiment. The $R m_{c}$ are measured in the case of iron propellers, and estimated using induction measurements for the inox propellers.

\section{Acknowledgements}

The numerical data in the paper are obtained through a collaboration with J-Ph. Laval, Y. Ponty, F. Daviaud, P. Blaineau and J-F. Pinton. The data from the von Karman water experiments have been obtained in Saclay in the group of F. Daviaud by A. Chiffaudel, F. Daviaud, L. Marié, F. Ravelet, R. Monchaux, P. Diribarne. The data from von Karman sodium experiment have been obtained in Cadarache by the VKS collaboration (CEA Saclay : S. Aumaitre, A. Chiffaudel, F. Daviaud, B. Dubrulle, C. Gasquet, L. Marie, R. Monchaux, V. Padilla and F. Ravelet; ENS Paris : M. Berhanu, S. Fauve, N. Mordant and F. Pétrélis; ENS Lyon : M. Bourgoin, Ph. Odier, J-F. Pinton, N. Plihon, R. Volk). The data interpretation is personal and does not engage the responsibility of any of these people.

\section{References}

Aumaitre, S., Pétrélis, F. \& Mallick, K., 2005, Phys. Rev. Lett., 95,064101

Avalos-Zuniga, R., Plunian, F. \& Gailitis, A., 2003, PRE, 68, 066307

Berhanu, M., Monchaux, R., Fauve, S., Mordant, N., Pétrélis, F., Chiffaudel, A., Daviaud, F., Dubrulle, B., Marié, F. Ravelet, L., Bourgoin, M., Odier, Ph., Pinton, J.-F., \& Volk, R. 2007, Eur. Phys. Rev. Lett., 77, 59001

Boldyrev, S. 2001, Astrophys. J., 562, 1081

Dubrulle, B. \& Frisch, U. 1991, Phys. Rev. A, 43, 5355 
Dubrulle, B., Blaineau, P., Mafra Lopez, O., Daviaud, F., Laval, J-P., \& Dolganov, R., 2007, New J. Phys., 9, 308

Krause, F. \& Rädler, K.-H., Mean field MHD and dynamo theory, 1980, Pergamon press.

Laguerre, R., Nore, C., Leorat, J., \& Guermond, J-L., 2006, C. R. Mécanique, 334, 593

Laval, J.-P., Blaineau, P., Leprovost, N., Dubrulle, B., Daviaud, F., 2006, Phys. Rev. Let., 96, 204503

Leprovost, N. \& Dubrulle, B., 2005, Eur. Phys. J. B, 44, 395

Moffatt, H. K., 1978 Magnetic field generation in electrically conducting fluids (Cambridge University Press).

Monchaux, R., Berhanu, M., Bourgoin, M., Odier, Ph., Moulin, M., Pinton, J.-F., Volk, R., Fauve, S., Mordant, N., Pétrélis, F., Chiffaudel, A., Daviaud, F., Dubrulle, B., Gasquet, C., Marié, L. \& Ravelet, F. 2007, Phys. Rev. Lett., 98, 044502

Monchaux, Romain, Berhanu, Michael, Aumaitre, Sebastien, Chiffaudel, Arnaud, Daviaud, Francois, Dubrulle, Berengere, Florent, Stephan Ravelet, Nicolas, Fauve, Francois Mordant, Mickael Petrelis, Philippe, Bourgoin, Jean-Francois Pinton, Odier, Plihon,Nicolas, \& Volk, Romain, 2009, Phys. Fluids, 21, 035108

Pétrélis, F., 2002, PhD Thesis Paris VI

Ponty, Y., Mininni, P. D., Pinton, J.-F., Politano, H., \& Pouquet, A. 2005, Phys. Rev. Lett., 94, 164512

Ponty, Y., Mininni, P. D., Pinton, J.-F., Politano, H. \& Pouquet, A. 2007, New J. Phys., 9, 296

Ravelet, F., Chiffaudel, A., Daviaud, F. \& Leorat, J. 2005, Phys. Fluids, 17, 17104

Ravelet, F., Berhanu, M., Monchaux, R., Aumaitre, S., Chiffaudel, A., Daviaud, F., Dubrulle, B., Bourgoin, M., Odier, Ph., Plihon, N., Pinton, J.-F., Volk, R., Fauve, S., Mordant, N., \& Petrelis, F., 2008,Phys. Rev. Lett., 101, 074502

Stefani, F., Xu, M., Gerbeth, G., Ravelet, F., Chiffaudel, A., Daviaud, F., \& Leorat, J., 2006, Eur. J. Mech. B, 25, 894

Volk, R., Ravelet, F., Monchaux, R., Berhanu, M., Chiffaudel, A., Daviaud, F., Fauve, S., Mordant, N., Odier, Ph., Pétrélis, F. \& Pinton, J.-F., 2006, Phys. Rev. Lett., 97, 074501

\section{Discussion}

E. Ntormousi: How far are your experiments from similarity to solar dynamo?

B. Dubrulle: Our device is cylindrical and not spherical (Smile). The energy source is through impellers rather than convection. But apart from that, we have regimes with periodical or aperiodical dynamos, exactly like in stars. So we think we may gain a lot of insight on the solar dynamo from studying our device.

C. Forest: What is the status of the soft iron propellors in getting or not dynamo action in VKS2?

B. Dubrulle: For the present time, we only get dynamo when at least one iron propellor is rotating. However, we have observed situations where dynamo depends on the sense of rotation of the iron impellers. This change mainly influences the sodium fluid properties. So this demonstrates that VKS is more than a pure "disk-dynamo" and that the fluid effect is very important. 